\title{
Intuitionistic Fuzzy Hyperhomomorphism and Intuitionistic Fuzzy Normal Subhypergroups
}

\author{
Karema S. Abdulmula, Abdul Razak Salleh \\ School of Mathematical Science, UKM, 43600 Bangi,Selangor Darul Ehsan, Malaysia \\ E-mail:karema1997saa@yahoo.com,aras@ukm.my
}

\begin{abstract}
The purpose of this paper is to introduce some basic concepts of intuitionistic fuzzy hyperalgebra. We continue our study of intuitionistic fuzzy hypergroups, by generalising the concept of fuzzy homomorphism and fuzzy normal subgroup based on fuzzy spaces to intuitionistic fuzzy hyperhomomorphism based on intuitionstic fuzzy spaces. We will introduce the notion of an intuitionistic fuzzy quotient hypergroup induced by an intuitionistic fuzzy normal subhypergroup under intuitionistic fuzzy hyperhomomorphism.
\end{abstract}

Keywords - Intuitionistic fuzzy hypergroup; Intuitionistic fuzzy normal subhypergroup; Intuitionistic fuzzy quotient hypergroup, Intuitionistic fuzzy hyperhomomorphism.

\section{INTRODUCTION}

The theory of intuitionistic fuzzy space is expected to play an important role in modern mathematics in general as it represents a generalization of fuzzy space. The notion of intuitionistic fuzzy space was first defined by Fathi and Salleh [1] as a generaliztion of Dib's [2] fuzzy space. After the concept of intuitionistic fuzzy space was introduced, several papers have been published by mathematicians to extend the classical mathematical concepts and intuitionistic fuzzy mathematical concepts to the case of intuitionistic fuzzy spaces. The dificulty in such generalizations lies in how to pick out the rational generalization from the large number of available approaches. The study of intuitionistic

Fuzzy hypergroups was stareted by Abdulmula and Salleh [3], by generalizing the notion of the fuzzy hypergroup based on fuzzy space by Fathi [4]. Abdul Razak Salleh in [5] introduced the notion of fuzzy normal subgroups continuing the work of Dib [2] on the theory of fuzzy groups. fuzzy normal subgroup by Dib and Hassan [6] also introduced in a similar manner to Abdul Razak Salleh, which depends on the concept of fuzzy space which serves as the concept of the universal set in the ordinary algebra. Thus Fathi and Salleh [7] continued the study of intuitionistic fuzzy groups by generalizing the notion of the fuzzy normal subgroup to the intuitionistic fuzzy normal subgroup case. Our interest in this paper is to continue the study of intuitionistic fuzzy hyper groups [7] by introducing the notion of an intuittionistic fuzzy quotient hypergroup induced by an intuitionistic fuzzy normal subhypergroup under intuitionistic fuzzy hyper homomorphisms based on intuitionistic fuzzy space as a generalisation of the intuitionistic fuzzy normal subgroup of [5] and prove the corresponding fundamental intuitionistic fuzzy homomorphism theorem.

\section{PRELIMINARIES}

Here we will recall some of the fundamental concepts and definitions required in the sequel.

Definition 2.1 (Abdulmula and Salleh [7]) Let $(H, I, I)$ be a nonempty intuitionistic fuzzy space. An intuitionistic fuzzy hyperstructure (intuitionistic fuzzy hypergroupoid), denoted by $\langle(H, I, I), \mathbf{F}\rangle$ is an intuitionistic fuzzy space together with an intuitionistic fuzzy function having onto comembership and cononmembership functions (referred as an intuitionistic fuzzy hyperoperation)

$F:(H, I, I) \times(H, I, I) \rightarrow P^{*}(H, I, I)$, where $P^{*}(H, I, I)$ denotes the set of all nonempty intuitionistic fuzzy subspaces of $(H, I, I)$ and $F=\left(F, f_{x y}, \bar{f}_{x y}\right)$ with

$F: H \times H \rightarrow P^{*}(H)$ and $\underline{f}_{x y}, \bar{f}_{x y}: I \times I \rightarrow I$

An intuitionistic fuzzy hyperoperation $\mathbf{F}=\left(F, \underline{f}_{x y}, \bar{f}_{x y}\right)$ on $(H, I, I)$ is said to be uniform if the associated comembership functions $\underline{f}_{x y}$ and cononmembership $\bar{f}_{x y}$ are identical, i.e., $f_{x y}=f$ and $\bar{f}_{x y}=f$ forall $x, y \in H$. A uniform intuitionistic fuzzy hyperstructure $\langle(H, I, I), \mathbf{F}\rangle$ is an intuitionistic 
fuzzy hyperstructure $\langle(H, I, I), \mathbf{F}\rangle$ with uniform intuitionistic fuzzy hyperoperation. Recall that the action of the intuitionistic fuzzy function $F=\left(F, f_{x y}, \bar{f}_{x y}\right)$ on intuitionistic fuzzy elements of the intuitionistic fuzzy space $(H, I, I)$ can be symbolized as follows

$(x, I, I) F(y, I, I)=F((x, I, I),(y, I, I))$

$$
\begin{aligned}
& =\left(F(x, y), \underline{f}_{x y}(I \times I), \bar{f}_{x y}(I \times I)\right) \\
& =(F(x, y), I, I) .
\end{aligned}
$$

Definition 2.2 (Abdulmula and Salleh [3]) An intuitionistic fuzzy hypergroup is an intuitionistic fuzzy hyperstrucure $\langle(H, I, I), \mathbf{F}\rangle$ satisfying the following axioms:

$$
\begin{aligned}
& \text { 1. }((x, I, I) \mathbf{F}(y, I, I)) \mathbf{F}(z, I, I)=(x, I, I) \mathbf{F}((y, I, I) \mathbf{F}(z, I, I)) \text {, } \\
& \quad \text { for all }(x, I, I),(y, I, I),(z, I, I) \text { in }(H, I, I), \\
& \text { 2. }(x, I, I) \mathbf{F}(H, I, I)=(H, I, I) \mathbf{F}(x, I, I)=(H, I, I), \\
& \quad \text { for all }(x, I, I) \text { in }(H, I, I) \text {. }
\end{aligned}
$$

Definition 2.3 (Fathi [1]) Let $X$ be a nonempty set. An intoitionistic fuzzy space (simply IFS) denoted by $(X, I, I)$ is the set of all ordered triples $(x, I, I)$ where

$(x, I, I)=\{(x, r, s): r, s \in I$ with $r+s \leq 1$ and $x \in X\}$. The ordered triple $(x, I, I)$ is called an intuitionistic fuzzy element of the intuitionistic fuzzy space $(X, I, I)$ and the condition $r, s \in I$ with $r+s \leq 1$ will be referred to as the intuitionistic condition".

Definition 2.4 (Fathi [1]) An intuitionistic fuzzy subspace $U$ of the intuitionistic fuzzy space $(X, I, I)$ is the collection of all ordered triples $\left(x, \underline{u}_{x}, \bar{u}_{x}\right)$, where $x \in U_{\circ}$ for some $U_{\circ} \in X$ and $\underline{u}_{x}, \bar{u}_{x}$ are a subsets of $I$, which contains at least one element beside the zero element. If it happens that $x \notin U$, then $\underline{u}_{x}=0$ and $\bar{u}_{x}=1$.

Definition 2.5 (Fathi [7]) Let $((G, I, I), F)$ and $\left(\left(G^{\prime}, I, I\right), H\right)$ be two intuitionistic fuzzy groups. An intuitionistic fuzzy homomorphism $\Phi$ of $((G, I, I), F)$ into $\left(\left(G^{\prime}, I, I\right), H\right)$ is an intuitionistic fuzzy function having onto comembership and cononmembership functions

\section{INTUITIONISTIC FUZZY NORMAL SUBHYPERGROUP}

Before defining the intuitionistic fuzzy normal subhyper gro-up we will introduce the notion of left (right) coset of intuiti-onistic fuzzy subhypergroup.

Definition 3.1 If $(U, \mathbf{F})$ where $U=\left\{\left(z, \underline{u}_{z}, \bar{u}_{z}\right): z \in U_{\circ}\right\}$ is an intuitionistic fuzzy subhypergroup of the intoitionistic fuzzy hypergroup $\langle(H, I, I), \mathbf{F}\rangle$ then for every intuitionistic fuzzy element $(x, I, I)$ of $(H, I, I)$, the intuitionistic fuzzy subspace defined by

$(x, I, I) U=(x, I, I) \mathbf{F} U=\left\{\left(x F z, \underline{f}_{x z}\left(I, \underline{u}_{z}\right), \bar{f}_{x z}\left(I, \bar{u}_{z}\right)\right)\right\}$ is called a left coset of the intuitionistic fuzzy subhypergroup $(U, \mathbf{F})$. Similarly, a right coset of the intuitionistic fuzzy subhypergroup $(U, \mathbf{F})$ is defined by the intuitionistic fuzzy subspace

$U(x, I, I)=U \mathbf{F}(x, I, I)=\left\{\left(z F x, f_{z x}\left(\underline{u}_{z}, I\right), \bar{f}_{z x}\left(\bar{u}_{z}, I\right)\right)\right\}$.

Definition 3.2 An intuitionistic fuzzy subhypergroup $U$ of the intuitionistic fuzzy hypergroup $\langle(H, I, I), \mathbf{F}\rangle$ is called an intuitionistic fuzzy normal subhypergroup if

(1) $\mathrm{U}$ is associative in $\langle(H, I, I), \mathbf{F}\rangle$,

(2) $(x, I, I) U=U(x, I, I)$ for all $x \in H$.

Example 3.3 Let $\mathrm{H}=\mathrm{S}_{3}$ be the set of all permutations on $\{1,2,3\}$. Define the intuitionistic fuzzy binary hyperoperation over the intuitionistic fuzzy space $(H, I, I)$ where $F$ is the set of ordinary composition of permutations and $\underline{f}_{x y}(r, s)=r \wedge s, \bar{f}_{x y}(r, s)=r \vee s$ for all $x, y \in H$. Consider the intuitionistic fuzzy subspace $U=\left\{\left(\epsilon,\left[0, \frac{1}{2}\right],\left[\frac{1}{2}, 1\right]\right),\left(\gamma,\left[0, \frac{1}{2}\right],\left[\frac{1}{2}, 1\right]\right)\right\}$ where $\epsilon$ denotes the identity permutation and $\epsilon=(12)$. One can easily investigate that $(U, \mathbf{F})$ is an associative intuitionistic fuzzy subgroup which is not normal.

Theorem 3.4 An intuitionistic fuzzy subhypergroup $(U, \mathbf{F})$ where $U=\left\{\left(z, \underline{u}_{z}, \bar{u}_{z}\right): z \in U_{\circ}\right\}$ of the intuitionistic fuzzy hypergroup $\langle(H, I, I), \mathbf{F}\rangle$ is an intuitionistic fuzzy normal subhypergroup iff

(i) $(U, F)$ is an ordinary normal subhypergroup of the ordinary hypergroup $(H, F)$.

(ii) $f_{x z}\left(I, \underline{u}_{z}\right)=f_{z^{\prime} x}\left(u_{z^{\prime}}, I\right), x F z=z^{\prime} F x$

where $x \in H$ and $z, z^{\prime} \in U_{\circ}$.

Theorem 3.5 Every intuitionistic fuzzy normal subhyper group $U$ of $\langle(H, I, I), \mathbf{F}\rangle$ defines an equivalence relation $\mathfrak{R}$ on the intuitionistic fuzzy space $(H, I, I)$ given by $(x, I, I) \Re(y, I, I) \Leftrightarrow(x, I, I) U=U(y, I, I)$.

The equivalence relation $\Re$ on the intuitionistic fuzzy space $(H, I, I)$ induces an equivalence relation on $H$ by the correspondence $(x, I, I) \leftrightarrow x$. That is,

$(x, I, I) \Re(y, I, I) \Leftrightarrow x \Re y$, which is equivalent to $x U_{\circ}=U_{\circ} y$.

Proof. The relation $(x, I, I) \Re(y, I, I)$ is an equivalence relation. It is followed from the normality of the intuitionistic fuzzy subhypergroup $U$. Moreover,

it is clear that $(x, I, I) U=U(y, I, I)$ leads to $x U_{\circ}=U_{\circ} y$, which defines an equivalence relation on $\mathrm{H}$. For every $x, y \in H$, and from the normality of the intuitionistic fuzzy subhypergroup $U=\left\{\left(z, \underline{u}_{z}, \bar{u}_{z}\right) ; z \in U_{O}\right\}$ we have 


$$
\begin{array}{r}
\left((x, I, I) \mathbf{F}\left(z_{1}, \underline{u}_{z_{1}}, \bar{u}_{z_{1}}\right)\right) \mathbf{F}\left((y, I, I) \mathbf{F}\left(z_{2}, \underline{u}_{z_{2}}, \bar{u}_{z_{2}}\right)\right) \\
=(x, I, I) \mathbf{F}\left(\left(z_{1}, \underline{u}_{z_{1}}, \bar{u}_{z_{1}}\right) \mathbf{F}(y, I, I)\right) \mathbf{F}\left(z_{2}, \underline{u}_{z_{2}}, \bar{u}_{z_{2}}\right) \\
=(x, I, I) \mathbf{F}\left((y, I, I) \mathbf{F}\left(z_{3}, \underline{u}_{z_{3}}, \bar{u}_{z_{3}}\right)\right) \mathbf{F}\left(z_{2}, \underline{u}_{z_{2}}, \bar{u}_{z_{2}}\right)
\end{array}
$$

$=(x F y, I, I) \mathbf{F}\left(z_{4}, \underline{u}_{z_{4}}, \bar{u}_{z_{4}}\right)$, where $z_{1}, z_{2}, z_{3}, z_{4} \in U o$.

where $z_{1}, z_{2}, z_{3}, z_{4} \in U o$.

Therefore, it follows that

$((x, I, I) U) \mathbf{F}((y, I, I) U)=(x F y, I, I) U$

Therefore, the intuitionistic fuzzy binary hyperoperation $F$ of the intuitionistic fuzzy hypergroup $\langle(H, I, I), \mathbf{F}\rangle$ induces a binary operation defined by the operation $(*)$ on the family of cosets of the intuitionistic fuzzy normal subhypergroup $U$. This family of cosets together with the induced binary operation forms an ordinary hypergroup called the factor hypergroup of $\langle(H, I, I), \mathbf{F}\rangle$ modulo $\mathrm{U}$ and denoted by $\langle(H, I, I), \mathbf{F}\rangle / U$. Now we define intuitionistic fuzzy normal subgroups induced by intuitionistic fuzzy subsets.

Definition 3.6 The intuitionistic fuzzy subset $A$ is said to be associateve in the intuitionistic fuzzy hypergroup $\langle(H, I, I), \mathbf{F}\rangle$ if the intuitionistic fuzzy subspace $H_{\circ}(A)=\{(x,\{0, \underline{A}(x)\},\{\bar{A}(x), 1\}): \underline{A}(x) \neq 0, \bar{A}(x) \neq 1\}$ is associative in $\langle(H, I, I), \mathbf{F}\rangle$. Then $H_{l u}(A)$ and $H_{u l}(A)$ are associative in $\langle(H, I, I), \mathbf{F}\rangle$.

Theorem 3.7 Let $A$ be an intuitionistic fuzzy subset of $H$. Then the intuitionistic fuzzy subspace $H_{\circ}(A),\left(H_{l u}(A)\right.$ or $\left.H_{u l}(A)\right)$, is an intuitionistic fuzzy normal subhypergroup of the intuitionistic fuzzy hypergroup $\langle(H, I, I), \mathbf{F}\rangle$, iff

1- $\left(A_{O}, F\right)$ is an ordinary normal subhypergroup of $(H, F)$

$2-\underline{f}_{x y}(1, \underline{A}(z))=\underline{f}_{z x}^{f} \cdot\left(\underline{A}^{\prime}\left(z^{\prime}\right), 1\right), \bar{f}_{x y}(1, \bar{A}(z))=\bar{f} \cdot\left(\bar{A}\left(z^{\prime}\right), 1\right)$

if $x F z=z^{\prime} F x$, for all $x \in H$ and $z, z \in A_{\circ}$.

3- $A$ is associative in $\langle(H, I, I), \mathbf{F}\rangle$

$$
f_{x y}(\underline{A}(x), \underline{A}(y))=\underline{A}(x F y), \bar{f}_{x y}(\bar{A}(x), \bar{A}(y))=\bar{A}(x F y),
$$

for all $x, y \in A_{0}$.

Now we introduce relationship between the introduced and the classical intuitionistic fuzzy normal subhypergroups as follows:

Theorem 3.8 Let $\langle(H, I, I), \mathbf{F}\rangle, \mathbf{F}=(F, f, \bar{f})$ be a uniform intuitionistic fuzzy hypergroup and let $\mathrm{f}$ be a $t$-norm. Then every intuitionistic fuzzy subset $A$ of $H$ which induces intuitionistic fuzzy normal subhypergroups is a classical intuitionistic fuzzy normal subhypergroup of $(H, \mathbf{F})$

Proof. If the intuitionistic fuzzy subsets $A$ induces intuitionistic fuzzy normal subhypergroups of the intuitionistic fuzzy normal hypergroup $\langle(H, I, I), \mathbf{F}\rangle$, then by Theorems 3.7 and $(*)$ the comembership and cononmembership functions $f, \bar{f}$ satisfies

$\underline{f}_{x y}(\underline{A}(x), \underline{A}(y))=\underline{A}(x F y)$ and $\bar{f}_{x y}(\bar{A}(x), \bar{A}(y))=\bar{A}(x F y)$,

for all $\underline{A}(x) \neq 0 \neq \underline{A}(y)$ and $\bar{A}(x) \neq 1 \neq \bar{A}(y)$. Therefore, if the intuitionistic fuzzy subset $A$ induces intuitionistic fuzzy normal subhypergroup, then $A$ satisfies the inequality $\underline{f}_{x y}(\underline{A}(x), \underline{A}(y)) \leq \underline{A}(x F y)$ and $\bar{f}_{x y}(\bar{A}(x), \bar{A}(y)) \geq \bar{A}(x F y)$,

for all $x, y \in H$. Therefore, $A$ is a classical intuitionistic fuzzy normal subhypergroup.

Theorem 3.9 Let $(U, \mathbf{F})$ be an ordinary normal subhypergroup of the ordinary hypergroup $(H, \mathbf{F})$. Then every intoitionistic fuzzy subset $A$ of $H$ for which $A_{\circ}=A$ induces an intuitionistic fuzzy normal subhypergroup of intuitionistic fuzzy hypergroup

$((H, I, I), \mathbf{G})$, where $\mathbf{G}=\left(G, \underline{g}_{x y}, \bar{g}_{x y}\right)$ and $G=F$, and $\underline{g}_{x y}, \bar{g}_{x y}$ are suitable comembership and cononmembership functions respectively.

Corollary 3.10 Every classical intuitionistic fuzzy normal subhypergroup $A$ of the hypergroup $(H, F)$ induces an intuitionistic fuzzy normal subhypergroups relative to some intuitionistic fuzzy hypergroup $\langle(H, I, I), \mathbf{F}\rangle$.

\section{INTUITIONISTIC FUZZY HYPERHOMOMORPHISMS}

In this section we introduce the notion of intuitionistic fuzzy hyperhomomorphism, intuitionistic fuzzy hyperisomorphism and intuitionistic fuzzy kernel also we study the action of intoitionistic fuzzy normal subhypergroups under intuitionstic fuzzy hyperhomomorphisms.

Definition 4.1 Let $\left\langle(H, I, I), F_{1}\right\rangle$ and $\left\langle\left(H^{\prime}, I, I\right), F_{2}\right\rangle$ be two intuitionistic fuzzy hypergroups. An intuitionistic fuzzy hyperhomomorphism $\Phi$ of $\left\langle(H, I, I), F_{1}\right\rangle$ into $\left\langle\left(H^{\prime}, I, I\right), F_{2}\right\rangle$ is an intuitionistic fuzzy function having onto comembership and cononmembership functions

$$
\begin{aligned}
& \Phi=\left(\phi, \underline{\varphi}_{x}, \bar{\varphi}_{x}\right):\left\langle(H, I, I), F_{1}\right\rangle \rightarrow\left\langle\left(H^{\prime}, I, I\right), F_{2}\right\rangle \\
& \text { such that } \Phi\left((x, I, I) F_{1}(y, I, I)\right)=(\phi(x), I, I) F_{2}(\phi(y), I, I) .
\end{aligned}
$$

The intuitionistic fuzzy hypergroups $\left\langle(H, I, I), F_{1}\right\rangle$ and $\left\langle\left(H^{\prime}, I, I\right), F_{2}\right\rangle$ are called intuitionistic fuzzy hyperhomomorphic hypergroups under the intuitionistic fuzzy hyperhomomorphism $\Phi$. 
If $\phi: H \rightarrow H^{\prime}$ is a bijection (one to one and onto) then $\Phi$ is called an intuitionistic fuzzy hyperisomorphism and the intuitionistic fuzzy hypergroups

$\left\langle(H, I, I), F_{1}\right\rangle,\left\langle\left(H^{\prime}, I, I\right), F_{2}\right\rangle$ are said to be intuitionistic fuzzy hyperisomorphic hypergroups and will be denoted by $\left\langle(H, I, I), F_{1}\right\rangle \cong\left\langle\left(H^{\prime}, I, I\right), F_{1}\right\rangle$.

From the above definition we can formulate the action of the intuitionistic fuzzy hyperhomomorphism

$\Phi=\left(\phi, \underline{\varphi}_{x}, \bar{\varphi}_{x}\right)$ of $\left\langle(H, I, I), F_{1}\right\rangle$ into $\left\langle\left(H^{\prime}, I, I\right), F_{2}\right\rangle$ for any two intuitionistic fuzzy elements $(x, I, I),(y, I, I) \in(H, I, I)$ as follow:

$\Phi\left((x, I, I) F_{1}(y, I, I)\right)=\Phi\left(\left(\left(x F_{1} y\right), I, I\right)\right)=\left(\phi(x) F_{2} \phi(y), I, I\right)$.

\section{Lemma 4.2 If}

$\Phi=\left(\phi, \underline{\varphi}_{x}, \bar{\varphi}_{x}\right):\left\langle(H, I, I), F_{1}\right\rangle \rightarrow\left\langle\left(H^{\prime}, I, I\right), F_{2}\right\rangle$

is an intuitionistic fuzzy hyperhomomorphism of intuitionistic fuzzy hypergroups

$\Phi=\left(\phi, \underline{\varphi}_{x}, \bar{\varphi}_{x}\right):\left\langle(H, I, I), F_{1}\right\rangle \rightarrow\left\langle\left(H^{\prime}, I, I\right), F_{2}\right\rangle$

having intuitionistic fuzzy identities $(e, I, I)$ and $\left(e^{\prime}, I, I\right)$

respectively then the following holds:

$1-\Phi((e, I, I))=(\phi(e), I, I)=\left(e^{\prime}, I, I\right)$,

$2-\Phi\left(\left(x^{-1}, I, I\right)\right)=\left(\Phi\left(\left(x^{-1}, I, I\right)\right)\right)^{-1}$.

Theorem 4.3 If $\Phi=\left(\phi, \underline{\varphi}_{x}, \bar{\varphi}_{x}\right)$ is an intuitionistic fuzzy hyperhomomorphism between the intuitionistic fuzzy hypergroups $\left\langle(H, I, I), F_{1}\right\rangle$ and $\left\langle\left(H^{\prime}, I, I\right), F_{2}\right\rangle$ then every associative intuitionistic fuzzy subhypergroup $U=\left\{\left(x, \underline{u}_{x}, \bar{u}_{x}\right): x \in U_{0}\right\}$ of $\left\langle(H, I, I), F_{1}\right\rangle$ is mapped under $\Phi$ to an associative intuitionistic fuzzy subhypergroup $\Phi(U)$ of $\left\langle(\phi(H), I, I), F_{2}\right\rangle$.

Proof Let $\Phi=\left(\phi, \underline{\varphi}_{x}, \bar{\varphi}_{x}\right)$ be an intuitionistic fuzzy hyperhomomorphism between $\left\langle(H, I, I), F_{1}\right\rangle$ and $\left\langle\left(H^{\prime}, I, I\right), F_{2}\right\rangle$ and let $U=\left\{\left(x, \underline{u}_{x}, \bar{u}_{x}\right): x \in U_{\circ}\right\}$ be any associative intuitionistic fuzzy subhypergroup of $\left\langle(H, I, I), F_{1}\right\rangle$ then

$$
\begin{aligned}
\left(\Phi(x) F_{2} \Phi(y)\right) F_{2} \Phi(z) & =\Phi\left(\left(x F_{1} y\right) F_{1} z\right) \\
& =\Phi\left(x F_{1}\left(y F_{1} z\right)\right)=\Phi(x) F_{2}\left(\Phi(y) F_{2} \Phi(z)\right)
\end{aligned}
$$

If $x$ (or $y$ or $z) \in(H, I, I)$ then $\Phi(x) \in(\phi(H), I, I)$ and if $x($ or $y$ or $z) \in U$ then $\Phi(x) \in \Phi(U)$. That is,

$\Phi(U)$ associative in $\left\langle(\phi(H), I, I), F_{2}\right\rangle$, whenever

$U$ associative in $\left\langle(H, I, I), F_{1}\right\rangle$.

Theorem 4.4 If $\Phi=\left(\phi, \underline{\varphi}_{x}, \bar{\varphi}_{x}\right)$ is an intuitionistic fuzzy hyperhomomorphism between the intuitionistic fuzzy hypergroups $\left\langle(H, I, I), F_{1}\right\rangle$ and $\left\langle\left(H^{\prime}, I, I\right), F_{2}\right\rangle$. Then every intuitionistic fuzzy normal subhypergroup
$U=\left\{\left(x, \underline{u}_{x}, \bar{u}_{x}\right): x \in U_{\circ}\right\}$ of $\left\langle(H, I, I), F_{1}\right\rangle$ is mapped under $\Phi$ to an intuitionistic fuzzy normal subhypergroup $\Phi(U)$ of $\left\langle(\phi(H), I, I), F_{2}\right\rangle$.

\section{Definition 4.5 Let}

$\Phi=\left(\phi, \underline{\varphi}_{x}, \bar{\varphi}_{x}\right):\left\langle(H, I, I), F_{1}\right\rangle \rightarrow\left\langle\left(H^{\prime}, I, I\right), F_{2}\right\rangle$ be an intoitionistic fuzzy hyperhomomorphism, de_ne:

(1) $\operatorname{keren} 1 \Phi=\left\{(x, I, I) \in\left\langle(H, I, I), F_{1}\right\rangle: \Phi((x, I, I))=\left(e^{\prime}, I, I\right)\right\}$, where $\left(e^{\prime}, I, I\right)$ is the intuitionistic fuzzy identity in $\left\langle\left(H^{\prime}, I, I\right), H\right\rangle$.

(2) image $\Phi=\left\{(y, I, I) \in\left\langle\left(H^{\prime}, I, I\right), F_{2}\right\rangle:(y, I, I)=\Phi((x, I, I))\right.$ for some $\left.(x, I, I) \in\left\langle(H, I, I), F_{1}\right)\right\}$.

Theorem 4.6 If

$\Phi=\left(\phi, \underline{\varphi}_{x}, \bar{\varphi}_{x}\right):\left\langle(H, I, I), F_{1}\right\rangle \rightarrow\left\langle\left(H^{\prime}, I, I\right), F_{2}\right\rangle$ be an intuitionistic fuzzy hyperhomomorphism, then

(1) $\operatorname{ker} \Phi$ define an intuitionistic fuzzy subhypergroups of $\left\langle(H, I, I), F_{1}\right\rangle$ and im $\Phi$ define an intuitionistic fuzzy subhypergroups of $\left\langle\left(H^{\prime}, I, I\right), F_{2}\right\rangle$.

(2) For any intuitionistic fuzzy elements $(x, I, I) \in \operatorname{ker} \Phi$ and $(y, I, I) \in\left\langle(H, I, I), F_{1}\right\rangle$ the following hold: $(y, I, I) F_{1}(x, I, I) F_{1}\left(y^{-1}, I, I\right) \in \operatorname{ker} \Phi$.

(3) $\Phi$ is one to one iff $\operatorname{ker} \Phi=\{(e, I, I)\}$.

Proof. (1) Straightforward by applying the definition of intoitionistic fuzzy subhypergroup or the related theorems.

(2) Using the properties of intuitionistic fuzzy hyperhomomorphism one can note that

$$
\begin{aligned}
\Phi\left((y, I, I) F_{1}(x, I, I) F_{1}\left(y^{-1}, I, I\right)\right) & =\Phi((y, I, I))\left(e^{\prime}, I, I\right) \Phi\left(\left(y^{-1}, I, I\right)\right) \\
& =\left(e^{\prime}, I, I\right)
\end{aligned}
$$

(3) Let be one to one and let $(x, I, I) \in \operatorname{ker} \Phi$, then $\Phi((x, I, I))=\left(e^{\prime}, I, I\right)$ and as $\Phi((e, I, I))=\left(e^{\prime}, I, I\right)$ we have $\Phi((x, I, I))=\Phi((e, I, I))$, , therefore using the fact that $\Phi$ is one to one we have $(x, I, I)=(e, I, I)$ and thus $\operatorname{ker} \Phi=\{(e, I, I)\}$. Conversely if $\operatorname{ker} \Phi=\{(e, I, I)\}$ we assume $\Phi((x, I, I))=\Phi((y, I, I))$ then we have $\Phi((x, I, I)) F_{1}(\Phi((y, I, I)))^{-1}=\left(e^{\prime}, I, I\right)$. Therefore $\Phi\left((x, I, I) F_{1}\left(y^{-1}, I, I\right)\right)=\left(e^{\prime}, I, I\right)$, which yields $(x, I, I) F_{1}\left(y^{-1}, I, I\right) \in \operatorname{ker} \Phi$. .

That is, $(x, I, I) F_{1}\left(y^{-1}, I, I\right)=(e, I, I)$ or $(x, I, I)=(y, I, I)$.

\section{Definition 4.7 Let}

$\Phi=\left(\phi, \underline{\varphi}_{x}, \bar{\varphi}_{x}\right):\left\langle(H, I, I), F_{1}\right\rangle \rightarrow\left\langle\left(H^{\prime}, I, I\right), F_{2}\right\rangle$ be an intuittionistic fuzzy hyperhomomorphism. kerenl $\Phi$ is called a strong intuitionistic fuzzy kernel (denoted by Sker $\Phi$ ) of the 
intuitionistic fuzzy hyperhomomorphism

$\Phi$ if $\operatorname{keren} l \Phi=\left\{(x, I, I) \in\left\langle(H, I, I), F_{1}\right\rangle: \Phi((x, I, I))=\left(e^{\prime}, I, I\right)\right\}$ define an associative intuitionistic fuzzy subhypergroup of $\left\langle(H, I, I), F_{1}\right\rangle$.

Corollary 4.8 An intuitionistic fuzzy kernel of the uniform intuitionistic fuzzy hyperhomomorphism $\Phi=\left(\phi, \underline{\varphi}_{x}, \bar{\varphi}_{x}\right)$ is a strong intuitionistic fuzzy kernel if $\underline{\varphi}_{x}$ and $\bar{\varphi}_{x}$ are $t$-norm functions.

\section{INTUITIONISTIC FUZZY QUOTIENT HYPERGROUPS}

In order to construct the de nition of intuitionistic fuzzy quotient hypergroup first we will define the addition of intuitionistic fuzzy subhypergroups $\langle U, \mathbf{F}\rangle$ and $\left\langle U^{\prime}, \mathbf{F}\right\rangle$ of the intuitionistic fuzzy hypergroup $\langle(H, I, I), \mathbf{F}\rangle$ as follow:

$\langle U, \mathbf{F}\rangle\left\langle U^{\prime}, \mathbf{F}\right\rangle=\left\{(u, I, I) F\left(u^{\prime}, I, I\right):(u, I, I) \in U\right.$ and $\left.\left(u^{\prime}, I, I\right) \in U^{\prime}\right\}$. Since both $\langle U, \mathbf{F}\rangle$ and $\left\langle U^{\prime}, \mathbf{F}\right\rangle$ are intuitionistic fuzzy sub hypergroups of $\langle(H, I, I), \mathbf{F}\rangle$. Then their addition still an intuitionistic fuzzy subhypergroup of. Moreover this addition is associative since the intuitionistic fuzzy binary hyperoperation $F$ is associative.

Consider the intuitionistic fuzzy normal subhypergroup $\langle U, \mathbf{F}\rangle$ where $U=\left\{\left(z, \underline{u}_{z}, \bar{u}_{z}\right): z \in U_{\circ}\right\}$ of the intuitionistic fuzzy hypergroup $\langle(H, I, I), \mathbf{F}\rangle$. Define the set $H / U$ to be the set of all intuitionistic fuzzy left cosets of $U$ in, i.e., $H / U=\{(x, I, I) U: x \in(H, I, I)\}$,

then we have the following theorem which is a fundamental tool for the construction of a new intuitionistic fuzzy hypergroup from a given intuitionistic fuzzy hypergroup using intuitionistic fuzzy normal subhypergroup.

Theorem 5.1 Given the intuitionistic fuzzy normal subhypergroup $\langle U, \mathbf{F}\rangle$ of the intuitionistic fuzzy hypergroup $\langle(H, I, I), \mathbf{F}\rangle$. The set is an intuitionistic fuzzy hypergroup under the addition hyperoperation given by

$$
(x, I, I) U(y, I, I) U=((x, I, I) F(y, I, I)) U \text {, }
$$

for all $(x, I, I),(y, I, I) \in H$

Proof From associativity we have:

$$
\begin{aligned}
(x, I, I) U(y, I, I) U & =(x, I, I)(U(y, I, I)) U \\
& =(x, I, I)((y, I, I) U) U \\
& =((x, I, I) F(y, I, I)) U U \\
& =((x, I, I) F(y, I, I)) U
\end{aligned}
$$

for which intuitionistic fuzzy normality of $U$ ensures the equality $(y, I, I) U=U(y, I, I)$ while $U U=U$ is clear because $U$ is originally an intuitionistic fuzzy subhypergroup. Thus the addition of two intuitionistic fuzzy cosets of $U$ is again an intuitionistic fuzzy coset of $U$, and so an operation on $H / U$ has been defined. Since the addition hyperoperation is associative, equality $X(Y Z)=(X Y) Z$ holds where $X, \quad Y$ and $Z$ are intuitionistic fuzzy subhypergroups of $\langle(H, I, I), \mathbf{F}\rangle$, in particular, when $X, Y$ and $Z$ are intuitionistic fuzzy cosets of $U$, so that the hyperoperation on $H / U$ is associative. The intuitionistic fuzzy coset $(e, I, I) U=U$ will act as an identity element in $H / U$ for which

$$
\begin{aligned}
((e, I, I) U)((x, I, I) U) & =(e, I, I) F(x, I, I) U \\
& =(x, I, I) U \\
& =(x, I, I) F(e, I, I) U n \\
& =((x, I, I) U)((e, I, I) U) .
\end{aligned}
$$

Finally the intuitionistic fuzzy cosets $\left(x^{-1}, I, I\right) U$ will be the inverse of $(x, I, I) U$ since

$$
\begin{aligned}
\left(\left(x^{-1}, I, I\right) U\right)((x, I, I) U) & =\left(x^{-1}, I, I\right) F(x, I, I) U \\
& =(e, I, I) U=(x, I, I) F\left(x^{-1}, I, I\right) U .
\end{aligned}
$$

Therefore $H / U$ is an intuitionistic fuzzy hypergroup.

Lemma 5.2 Every intuitionistic fuzzy normal subhypergroup $\langle U, \mathbf{F}\rangle$ of an intuitionistic fuzzy hypergroup $\langle(H, I, I), \mathbf{F}\rangle$ is the strong intuitionistic fuzzy kernel of some intuitionistic fuzzy hyperhomomorphism.

Proof Define the intuitionistic fuzzy function

$$
\begin{aligned}
& \Phi=\left(\phi, \underline{\varphi}_{x}, \bar{\varphi}_{x}\right):\langle(H, I, I), \mathbf{F}\rangle \rightarrow H / U \\
& \text { by } \Phi((x, I, I))=(x, I, I) U
\end{aligned}
$$

( note that the hyperoperation over the intuitionistic fuzzy hypergroup $H / U$ is the addition hyperoperation). Now with help of this notation the equality $(x, I, I) U(y, I, I) U=(x, I, I) F(y, I, I) U$ can be rewrittenas :

$$
\Phi((x, I, I)) \Phi((x, I, I))=\Phi((x, I, I) F(y, I, I))
$$

Since $U$ is the intuitionistic fuzzy identity element in $H$ / $U$ we have:

$$
\begin{aligned}
& \operatorname{ker} \Phi=\{(x, I, I) \in\langle(H, I, I), F\rangle: \Phi((x, I, I))=U\} . \text { That is, } \\
& \operatorname{ker} \Phi=\{(x, I, I) \in\langle(H, I, I), F\rangle:(x, I, I) U=U\}=U .
\end{aligned}
$$

Since by our assumption that $U$ is an intuitionistic fuzzy normal subhypergroup we have $\operatorname{ker} \Phi$ is a strong intuitionistic fuzzy kernel, that is $\operatorname{ker} \Phi=\operatorname{Sker} \Phi$. Now we state and prove the fundamental theorem of intuitionistic fuzzy hypergroup hyperhomomorphism which relates the image of an intuitionistic fuzzy hypergroup $\langle(H, I, I), \mathbf{F}\rangle$ with an intuittionistic fuzzy quotient hypergroup of $\langle(H, I, I), \mathbf{F}\rangle$ in term of hyperisomorphic.

\section{Theorem 5.3 If}

$\Phi=\left(\phi, \underline{\varphi}_{x}, \bar{\varphi}_{x}\right):\langle(H, I, I), F\rangle \rightarrow\left\langle\left(H^{\prime}, I, I\right), H\right\rangle$ is an onto intuitionistic fuzzy hyperhomomorphism having a strong intuitionistic fuzzy kernel Sker $\Phi$, then $H / \operatorname{Sker} \Phi \cong H^{\prime}$. . 
Proof For simplicity let $U=\operatorname{Sker} \Phi$ and define an intuitionistic fuzzy function

$\Psi=\left(\psi, \underline{\psi}_{x}, \bar{\psi}_{x}\right): H / \operatorname{Sker} \Phi \rightarrow\left\langle\left(H^{\prime}, I, I\right), H\right\rangle$,

such that $\Psi((x, I, I) U)=\Phi((x, I, I))$.

Now we show that $\Psi$ is an intuitionistic fuzzy hyperisomorphism. That $\Psi$ is well defined follows by

$$
\begin{aligned}
(x, I, I) U=(y, I, I) U & \Rightarrow\left(y^{-1}, I, I\right) F(x, I, I) \in U=\operatorname{Sker} \Phi \\
& \Rightarrow \Phi\left(\left(y^{-1}, I, I\right) F(x, I, I)\right)=\left(e^{\prime}, I, I\right) \\
& \Rightarrow \Phi((y, I, I))^{-1} \Phi((x, I, I))=\left(e^{\prime}, I, I\right. \\
& \Rightarrow \Phi((x, I, I))=\Phi((y, I, I)) \\
& \Rightarrow \Psi((x, I, I) U)=\Psi((y, I, I) U) .
\end{aligned}
$$

Retracing the above step backward it is clear that $\Psi$ is one to on. Also as

$$
\begin{aligned}
\Psi((x, I, I) U(y, I, I) U) & =\Psi((x, I, I) F(y, I, I) U) \\
& =\Phi((x, I, I) F(y, I, I)) \\
& =\Phi((x, I, I)) \Phi((y, I, I)) \\
& =\Psi((x, I, I) U) \Psi((y, I, I) U) .
\end{aligned}
$$

proves that $\Psi$ is an intuitionistic fuzzy hyperhomomorphism. Finally we show that $\Psi$ is onto by considering any intuitionistic fuzzy element $\left(x^{\prime}, I, I\right) \in\left\langle\left(H^{\prime}, I, I\right), H\right\rangle$. Since by assumption $\Psi$ is onto there exist $(x, I, I) \in((H, I, I), F)$ such that $\Phi((x, I, I))=\left(x^{\prime}, I, I\right)$. Now $\Psi((x, I, I) U)=\Phi((x, I, I))=\left(x^{\prime}, I, I\right)$. Showing thereby that $(x, I, I) U$ is the required intuitionistic fuzzy preimage of $\left(x^{\prime}, I, I\right)$ under $\Psi$. Hence we have $\Psi$ is an intuitionistic fuzzy hyperisomorphism. Based on Corollary 4.8 if the intoitionistic fuzzy hyperhomomorphism $\Phi=\left(\phi, \underline{\varphi}_{x}, \bar{\varphi}_{x}\right)$ was uniform and $\underline{\varphi}_{x}, \bar{\varphi}_{x}$ were both a t-norm functions then we will guarantee the existence of a strong intuitionistic fuzzy kernel. Therefore the fundamental theorem of intuitionistic fuzzy hypergroups does not go in a parallel line to the ordinary fundamental theorem of hyper group hyperhomomorphism since in the ordinary case every kernel of a given hypergroup hyperhomomorphism is indeed a normal subhypergroup.

Corollary 5.4 Every classical intuitionistic fuzzy normal subhypergroup $A$ of hypergroup $\langle U, \mathbf{F}\rangle$ induces an intuitionistic fuzzy normal subhypergroups relative to some intuitionistic fuzzy hypergroup $\langle(H, I, I), \mathbf{F}\rangle$.

\section{CONCLUSION}

In this paper, we have generalized the study initiated about fuzzy hyperhomomorhisms of fuzzy groups to the context of intuitionistic fuzzy hyperhomomorphism of intuitionistic fuzzy hypergroups. We define the notion of an intuitionistic fuzzy hyperhomomorphizm using the notion of an intuitionistic fuzzy space .

\section{ACKNOWLEDGMENT}

The authors would like to acknowledge the financial support recei- ved from Universiti Kebangsaan Malaysia under the research grant UKM-ST-06-FRGS0104-2009

\section{REFERENCES}

[1] F. Mohammed, A. R. Salleh . On intuitionistic fuzzy spaces. Proceedings 16th National Symposium of Mathematical Sciences (SKSM-16), University Malaysia Terengganu, 2008.

[2] D. Kamal On fuzzy spaces and fuzzy group theory. Information Sciences 80: 253-282, 1994.

[3] K. S. Abdulmula \& A. R. Salleh. Intuitionistic fuzzy hyper groups. Proc. 2nd Int. Conf. Math. Sci: 218-225, 2010.

[4] F. Mohammed, Intuitionistic fuzzy algebra and fuzzy hyperalgebra. $\mathrm{PhD}$ thesis. Universiti Kebangsaan Malaysia, 2010.

[5] A. R. Salleh. Properties of fuzzy homomorphism of fuzzy groups (in Malay). Proc. 7th National Symposium of Mathematical Sciences. Institut . Teknologi Mara: 80: 346-351, 1996.

[6] D. Kamal, A. M. Hassan, The fuzzy normal subgroup theory. Fuzzy Sets and Sestems 98: 393-402, 1998.

[7] F. Mohammed, A. R. Salleh. Intutionistic fuzzy normal subgroups. International Journal of Fuzzy Logic and Intelligent Systems 10: 8288,2009 